\title{
Rétif de La Bretonne : un marginal traitant des marges sociales
}

Belkahla ILHEM

Faculté des Sciences Humaines et Sociales de Tunis/

Université Paris-Sorbonne (Paris IV)

$\mathrm{D}$ epuis quelques temps, les études sur la prostitution, un espace et une composante des marges sociales occupent les chercheurs en histoire, en philosophie, en médecine et en littérature. Classe inférieure et longtemps restée à l'écart, les prostituées sont généralement étudiées dans le rapport qu'elles entretiennent avec les normes sociales. Ce que nous interrogeons nous dans cet article, c'est leur marginalité, leur instabilité et leur pauvreté dans leur rapport avec l'espace géographique et commercial et les conditions sociales de l'époque. Cette perspective est légitime, nous semble-t-il, dans la mesure où l'histoire et la littérature de la France d'Ancien régime cessent de privilégier les premiers rôles et donnent la parole aux classes obscures. En même temps, étudier la prostitution à Paris au XVIII ${ }^{\mathrm{e}}$ siècle, c'est aborder un sujet historique et mythique car les romans faisant l'état des lieux encombre le terrain. Dans ce contexte, écrire sur les prostituées pour Rétif de La Bretonne ${ }^{1}$, c'est choisir leur camp, semble-t-il, surtout si l'on considère son Pornographe où il s'agit d'un projet de réforme sérieux pour les filles publiques.

Les textes de Rétif de La Bretonne, pourtant, n'appartenant pas à la « grande littérature » et qui, pour cette raison, sont souvent laissés de côté, nous donne une vision profonde, authentique et fort instructive sur le mondes des marges sociales et l'art de sa représentation. C'est à travers les textes de cet écrivain méconnu du grand public et longtemps laissé à 
la marge des critiques littéraires qui lui réserve un espace très timide dans leurs écrits, que va se former pour nous l'image de la vie débordante et singulière d'une classe marginale, qui a préoccupé les philosophes, les historiens, les historiographes et les écrivains depuis l'Antiquité, à savoir les prostituées. En effet, la production littéraire de Rétif, située dans une hiérarchie implicite des valeurs sociales et esthétiques offre de surcroît une ouverture sur les caractères constitutifs de l'identité marginale, notamment celle de la prostituée.

Alors que jusqu'au début du $\mathrm{XVI}^{\mathrm{e}}$ siècle, la prostitution est restée tolérée, sous le contrôle de L'État pendant l'Antiquité et organisée par les autorités municipales au Moyen Âge, on assiste à partir des années 1520, en liaison avec les réformes religieuses mais surtout avec l'apparition de la syphilis à un regard différent sur ce fléau social. Dès lors, se dessine, de la part des gouvernements qui se succèdent et des lois décrétées, un rejet de la prostitution et des prostituées et un effort de contrôler et d'encadrer ce domaine au nom de la morale mais surtout de la santé publique. Ainsi, dès le milieu du XVI ${ }^{\mathrm{e}}$ siècle, des mesures sont décrétées contre les prostituées. D'abord, « [e]n 1542, le Sénat de Venise leur interdit d'avoir chez elles des garnitures de soie, des tapisseries et des meubles précieux (. . .) à Florence et à Venise on tente de leur imposer un vêtement plus sombre que celui des autre femmes $»^{2}$. Ensuite, en 1560, un édit connu sous le nom d'ordonnance d'Orléans interdit « les bordeaux ». Suite à cet édit les maisons publiques municipales en France sont fermées et les entremetteurs poursuivis.

Cependant, et à cause de la persistance de la prostitution, clandestine cette fois-ci,

Une nouvelle étape est franchie au XVII ${ }^{e}$ siècle avec l'instauration d'une politique plus vaste, « le renfermement », qui vise aussi bien les mendiants, les fainéants, les vagabonds, les gens sans aveu que les débauchés. Ces derniers sont passibles de l'enfermement dans les hôpitaux généraux, qui fonctionnent à la fois comme des hôpitaux, des hospices et des maisons de force ${ }^{3}$.

Ainsi à Paris, les prostituées arrêtées sur la voie publique sont emprisonnées à la Salpêtrière, ouverte en 1656. A la fin du XVIII ${ }^{\mathrm{e}}$ siècle, Louis-Sébastien Mercier décrit ainsi leur arrivée dans ce lieu :

On les fait monter dans un long chariot, qui n'est pas couvert. Elles sont toutes debout et pressées. L'une pleure, l'autre gémit ; 
celle-ci se cache le visage ; les plus effrontées soutiennent les regards de la populace qui les apostrophe ; elles ripostent indécemment et bravent les huées qui s'élèvent sur leur passage. Ce char scandaleux traverse une partie de la ville en plein jour ; les propos que cette marche occasionne sont encore une atteinte à l'honnêteté publique ${ }^{4}$

Les mesures de la marginalisation et de mise à l'écart sont de plus en plus mises en place. Elles se traduisent dans l'isolement des prostituées dans des quartiers séparés ou en dehors de la ville. A Paris, tout un appareil policier est mis en place pour combattre la prostitution et empêcher son augmentation. " $\mathrm{Au} \mathrm{XVIII}$ " siècle, à Paris, on compte quelques 20000 femmes s'adonnant à la prostitution, soit 13 mille des Parisiennes, sans compter les occasionnelles $\nu^{5}$. L'insuffisance des revenus du travail sont souvent données comme facteurs explicatifs de la prostitution par les prostituées quand elles ont affaire à la police. Mais au-delà des explications, Rétif de La Bretonne montre dans tout ce qu'il a écrit sur la prostitution, qu'outre la misère, il est difficile d'évaluer le rôle qu'a pu jouer la prostitution pour ces malheureuses. En effet, il est certain que les trois quarts d'entre elles se rangent dans des groupes d'immergées issues d'un milieu campagnarde et qui, pour lesquels se pose le problème de la subsistance en ville. Le témoignage de Rétif sur le monde de la prostitution et les prostituées est le produit d'une recherche en profondeur. En effet, ses romans mettent en scène des personnages qui ont laissé des traces dans ce que nous pensons être des histoires vraies. Sa reconstitution du cercle intime et corrompu de la prostitution dans la ville de Paris, des causes, des effets pernicieux et de son durable impact sur les victimes d'aujourd'hui et d'autrefois ne peuvent émaner que d'un connaisseur de cause : un client assidu des prostituées. Ainsi il déclare : « Je faisais alors la seconde édition de mon Pornographe, et je voyais beaucoup de filles, pour connaître à fond cet état vil ... Lecteur, je ne suis pas un homme ordinaire : rappelez-vous que je suis auteur et qu'un auteur tel que moi doit, comme le médecin, essayer les poisons pour vous en préserver $\rangle^{6}$. L'œuvre de Rétif reconstitue toute une philosophie de la classe populaire marginale de l'époque. En effet, la description sociale des milieux populaires, des lieux restés à la périphérie, laisse penser qu'une grande partie de l'œuvre de Rétif pourrait être envisagée comme une forme d'écriture sociologique concernée par la représentation des marges sociales. Cependant, nous estimons que sans être de la sociologie, l'écriture de Rétif partage un champ et des techniques avec les chercheurs du terrain, en particulier par ses qualités d'observateur 
obsessif et ses descriptions maniaques. Il transmet une perception des choses telles qu'elles avaient existé avec une implication de soi qui fait de lui un observateur participant. La description par James Clifford de 《l'anthropologue voyageur $»^{7}$ pourrait très bien servir à caractériser les romans de Rétif. En effet, son parcours continu est guidé par la curiosité qui pourrait être celle d'un voyageur-reporter parfois, d'un chercheur mais surtout d'un preneur de notes. Ses récits détaillés des conversations avec les prostituées, notamment dans Le Palais-Royal, et les descriptions minutieuses des lieux en témoignent fort. Ces éléments centraux de son style semblent l'assimiler instantanément à un anthropologue. Ainsi, la conception rétivienne de la prostitution se veut à dominante documentaire, nous semble-t-il.

Il y a plus de quarante ans, Michel Foucault publiait l'Histoire de la Folie à l'âge classique, qui mettait en valeur la grande opération du renfermement des marginaux dans les hôpitaux généraux. Il révélait ainsi comment une société désignait autrement et séparait ce qu'elle ne pouvait plus supporter. C'est ainsi qu'à partir du XVIII ${ }^{\mathrm{e}}$ siècle, la représentation de la marginalité et des marginaux notamment les prostituées change dans l'imagination et dans la raison, en même temps que les conditions économiques, sociales et politiques se transforment. Pour Rétif de La Bretonne, toute société engendre des prostituées, et même les secrète. Elle en a besoin pour vivre. Bien sûr, les prostituées constituent un danger parce qu'elles ne respectent pas les valeurs essentielles de la société civile. Mais en même temps, elles rendent à la société un immense service en lui permettant de rappeler les éléments majeurs autour desquels s'établit l'ordre général.

Depuis l'Antiquité, toute société tente de circonscrire ses prostituées dans des limites en quantités et en qualités acceptables. Elle se livre alors aux jeux cruels et simultanés de l'assistance et la répression. Si les prostituées mettent l'ordre social en péril, la société tente de les récupérer, soit en leur donnant les moyens d'une existence « normale », soit en leur accordant un statut qu'elle ne leur reconnaissait pas jusqu'alors. Mais si la tentation d'intégration échoue, elle les condamne et par là les exclut, leur assignant la résidence dans un espace où il n'y a pas de contact avec le monde extérieur. Ainsi, au siècle des Lumières on enfermait les filles publiques à la Salpêtrière ou bien on les embarquait pour le Mississipi où la plupart périrent en route.

Chassée de la vie sociale, exclue et cachée aux yeux du public, la prostituée finirait enfermée à la Salpêtrière ou un personnage littéraire. Cette ambigüité débouche, à la fin du XVIII', sur un projet que l'on pourrait considérer comme un essai de synthèse des deux résultats qui préfigurent le 
règlementarisme du XIX ${ }^{\mathrm{e}}$ siècle : les Parthénions de Rétif de La Bretonne. Rétif, féministe avant-gardiste, voulait que ces malheureuses marginales échappent à l'enfermement définitif et à la fatalité. D'où l'idée des Parthénions, un asile inviolable pour les prostituées qui pourraient cependant le quitter en cas de repentie sincère. Ce sont des maisons parfaitement hiérarchisées et gérées uniquement par des femmes. Il s'agit d'un univers idéal, en effet, au désordre reproché à la prostitution, Rétif oppose un univers totalement ordonné, un ordre qu'on peut qualifier de cartésien.

Ce projet de réforme vise à réhabiliter les prostituées longtemps humiliées, maltraitées et méprisées et qui sont avant tout des êtres humains à part entière dignes de respect Dans Le Paysan perverti et La Paysanne pervertie, Rétif a laissé un tableau tragique de la condition de la prostituée ; et il était très conscient de l'acuité du problème qu'il écrit Le Pornographe pour proposer ses fameux Parthénions. Par ailleurs, au tournant du siècle des Lumières, la prostitution apparaît comme la composante la plus dominante des marges sociales qui permet d'interroger les liens entre commerce et sexualité ou encore métier et sexualité. Un commerce prospère dans « le point le plus chaud» de Paris à savoir le Palais-Royal, mais qui ne conduit guère à la prospérité de celles qui le pratiquent nous semble-t-il. Historiens et critiques littéraires ont du mal à classer ces femmes à mi-chemin entre ouvrières et prostituées ou encore commerce et prostitution. Dans ce contexte, l'écriture de Rétif de La Bretonne semble traduire cette angoisse générale de déclassement de ces femmes. Les écrits de ce dernier explicitent ce rapport ternaire d'apparence complexe qui trouve ses lettres de noblesse dans les conditions de vie très difficile d'un Ancien Régime agonisant.

En effet, la veille de la révolution, la misère et la difficulté de la vie dans la capitale imposent à une grande majorité des femmes roturière un modèle de vie qui ne s'accorde pas souvent avec la morale. Pour ces dernières, la prostitution est souvent le refuge, un refuge pour ceux que la vie oblige à perdre l'essentiel : le droit de vivre dignement. Dans ce contexte, Érica-Marie Benabou a fait une étude socio-professionnelle des prisonnières de Saint-Martin, prison où on détenait les filles publiques au XVIII ${ }^{e}$ siècle et a remarqué qu'une grande partie des filles prisonnières pratiquait des métiers féminins : les métiers du textile et leurs dérivés, la marchandise de mode. Cette étude semble aller en parallèle avec le recueil des Contemporaines du Commun de Rétif où il étale une galerie de gens de commerce ou de profession « où les sujets sont infiniment nombreux, que l'insuffisance de leur gain réduit à mener la vie la plus misérable, ou à manquer d'honnêteté. Telles sont les filles couturières, tapissières, etc. $\rangle^{8}$ 
C'est ainsi que dans "La fille sauvée », une nouvelle des Contemporaines du commun, Rétif raconte comment il a sauvé une fille qui était sur le point de déchoir dans la débauche : il s'agit d'une jeune ouvrière « jolie et naïve ». L'insuffisance de son salaire l'a obligée à céder aux insistances de ses deux compagnes pour venir la nuit avec elles chercher un gain facile.

En effet, de toutes les causes de la prostitution, particulièrement à Paris, il n'en est pas de plus active que le défaut de travail et la misère, suite inévitable de salaires insuffisants car que gagnent les coutrières, les boulangères ou les ravaudeuses ... Indigné de leur situation Parent-Duchâtelet, un médecin hygiéniste français contemporain de Rétif pousse un cri d'indignation et de colère

Que l'on compare le gain des plus habiles, avec celui qui peuvent faire celles qui n'ont que des talents médiocres, et l'on verra s'il est possible à ces dernières de se procurer le strict nécessaire ; que l'on compare surtout le prix de leur travail avec celui de leur déshonneur, et l'on cessera d'être surpris d'en voir un si grand nombre tomber dans un désordre pour ainsi dire inévitable ${ }^{9}$

Les Contemporaines du commun semblent illustrer fortement ce point de vue, Rétif y parle par exemple des gazières qui fabriquent la gaze destinée aux plus somptueuses robes et qui sont très peu payées « c'est le plus pauvre des métiers que celui qui pare l'opulence $»^{10}$. Aussi sont-elles " presque toutes libertines, ou prête dès qu'il se présente un tentateur $»^{11}$. Rétif préconise l'augmentation de leurs salaires pour les soustraire de la corruption. De même pour les ouvrières en mode, Rétif pense qu'il est impossible de vivre avec des salaires de neuf sous par jour ; elles n'ont donc d'autres sources que de se vendre, à moins de survivre en mangeant de la nourriture frelatée.

Dans la nouvelle «Les huit petites-marchandes du Boulevard» une nouvelle des Contemporaines du commun, Rétif met en avant des petits métiers, qui, à cause de leur pauvre revenu présentent un grand risque pour la vertu des filles, nous citons dans l'ordre ces métiers : la petite mercière, la petite épinglière, la petite-éventailliste, la petite bouquetière, la petitebonnetière-en-mode, la petite poudrière-pommadière, la petite gaufrière et la petite vendeuse de fruits. ${ }^{12}$ Parmi ces métiers, " les bouquetières sont particulièrement menacées par les dangers de la prostitution : c'est du moins ce que suggère un opuscule de $1779 \gg^{13}$. La petite bouquetière de Rétif raconte ce qu'elle subissait dans son travail : « [...] quand j'vas à la portière des carrosses porter des bouquets aux Messieurs [...] c'est 
qu'en-montant su' l'montoir, l'un vous prend la joue, l'aute l'menton, l'aute un-peu-pûs-bas [...] $»^{14}$. La nature de ce métier oblige ces femmes misérables à céder à ces tentations viles pour ne pas perdre leur travail.

Une autre grande classe des travailleuses fournissait au XVIII ${ }^{\mathrm{e}}$ siècle une grande majorité des prostituées, cette classe que disait Rétif « la dernière classe du peuple des grandes villes » est celle des revendeuses, héroïnes d'un grand nombre de nouvelles dans Les Contemporaines $d u$ commun. Ces filles se divisent selon Rétif en deux types : les revendeuses séduisantes dont la misère est attendrissante comme la jolie fruitière et les revendeuses à la mise ${ }^{15}$ grossière et sans attrait comme la crieuse des vieux chapeaux. L'itinéraire de ces dernières vers la prostitution est facile et automatique. Benabou explique « $34,6 \%$ des inscrites régulièrement vivent dans les faubourgs, souvent en garni, c'est-à-dire loin des lieux d'achat et de revente qui se situent au cœur de Paris, aux Halles, place du Louvre, marché du Palais -Royal (...). Autant d'emplacement proches des lieux de raccroc ou se confondant avec eux $»^{16}$. Ces « crieuses » étaient hautement surveillées au XVIII" siècle. En effet, « les revendeuses doivent tenir un livre de police, contrôlé tous les mois par le commissaire du quartier, où sont consignés les achats, les prix pratiqués, les noms de vendeurs. Dans le même temps, ces accointances de la prostitution facilitent la récupération policière des crieuses $\gg{ }^{17}$.

Un autre métier féminin de cette période semble être en rapport étroit avec la prostitution, vu que c'est un métier très pénible et d'un très petit revenu à savoir les blanchisseuses. Ces femmes constituaient selon Benabou 14,5\% soit 300 des femmes enfermées à Saint-Martin pour débauche. Cette classe de travailleuses n'a pas échappé aussi aux représentations de Rétif, Javote une blanchisseuse était l'une des héroïnes de la nouvelle « Les huit petites-marchandes » qui se présentait ainsi « J'sis fille d'une blanchisseuse de la rue Perdue. J'avais tant d'peine dans ma jeunesse, qu'ça-f'sait-pitié. Aussi, quand j'passais chargée d'linge pour aler au bateau, ceux qui m'rencontraient, m'disaient : Monguieu ! quée dommage ! La jolie enfant ! (..) ${{ }^{18}}^{18}$, nous apprenons à la fin de la nouvelle que cette même Javote est devenue Zaïre, une belle prostituée qui logeait dans une maison publique.

Ainsi se dessine une sociologie de la corruption, liée à la misère et aux métiers dus essentiellement à la mal organisation sociale. Et Rétif a bien conscience que la morale est un mot vide de sens pour tous ceux qui vivent misérablement. Ils ne peuvent avoir que des idées rampantes comme « la jolie lunetière », qui se méprise elle-même et pense n'avoir rien à ménager (nouvelle ${ }^{\circ} \mathrm{XXIII}, \mathrm{p} .180$ ) : « la misère aigrit, elle fait haïr l'aménité » (nouvelle ${ }^{\circ} \mathrm{XXIX}, \mathrm{p} .107$ ). 
Le problème moral de la prostitution se trouve déplacé sur le terrain de l'économie et de la politique. Rétif, incrimine ici l'inégalité des conditions sociales et les progrès des Lumières. Il souligne que les prostituées traduisent l'hypocrisie d'une société qui leur refuse éducation et moyens honnêtes de gagner leur vie et les rejette au moindre faux pas.

Benabou note qu'une grande majorité des filles de la prison de SaintMartin appartiennent à des métiers comme des couturières, lingères, bouquetières, ou les petits commerces alimentaires soit encore dans le blanchissage du linge, des métiers où la femme tient un monopole de fait. Or, les métiers spécifiquement féminins occupent au XVIII ${ }^{\mathrm{e}}$ siècle le bas de l'échelle dans la hiérarchie générale des salaires. Dans ces conditions, un salaire de 15 sous par jour et souvent bien moins ne saurait suffire à nourrir, loger et vêtir une femme. Les jeunes d'entre elles gagnent encore moins, ainsi nous apprenons dans un procès de mœurs qu'une « ouvrière à l'année » débute pour 24 écus de gages, chez une maîtresse couturière, une somme qui ne fait même pas une ivre de pain pour une semaine, mais sa maîtresse lui fait miroiter six francs pour consentir à se prostituer. ${ }^{19}$

Et à Rétif de se demander comment un salaire pareil permet-il de vivre dignement. Il en donne un exemple fort révélateur dans ses Contemporaines d'une travailleuse d'aiguille, Babet, qui gagne neuf sous par jour et en dépense trois pour le pain, trois pour le loyer et trois pour une soupe d'auberge et dort en fin de journée sans aucun sous.

Dans ce contexte, l'historienne Érica-Marie Benabou affirme que

C'est d'abord le problème du pain quotidien, ou du loyer, que vient résoudre la prostitution. Cet aspect de revenu complémentaire a été perçu par plusieurs écrivains à la fin de l'Ancien-Régime. Parmi eux, outre Restif, Bérenger, pourtant peu indulgent et moralisateur, indigné de la multiplication des femmes publiques, les considère néanmoins comme « souvent plus affamées que libertines $»{ }^{20}$

Une autre raison dans ce même contexte semble renforcer cette idée, c'est l'irrégularité dans les métiers féminins saisonniers qui connaissent les mortes-saisons : «Ainsi le plein été, avec ses couturières, ses boutiquières, ses domestiques désœuvrées, moment record d'arrestations de filles $»^{21}$. Rétif écrit à ce propos dans son Pornographe: «Les filles de moyenne vertu qui ne se prostituent que par intérim, dans de mortes-saisons pour leurs métiers, et dans la seule de subvenir à des besoins pressants $\rrbracket^{22}$. L'historien Vincent Milliot affirme dans ce même ordre d'idées que : «La 
tentation est forte avec de très faibles rémunérations ou lorsque la crise frappe les ouvrières de l'artisanat de luxe $»^{23}$.

Dans ce même contexte les travaux de l'historien Milliot viennent consolider les constatations et les témoignages de Rétif et les recherches de Benabou. En effet, ce dernier pense aussi que les trois-quarts des filles de joies appartiennent au monde du travail « $54 \%$ des prisonnièresde l'Hôpital Saint-Martin exercent une activité artisanale avec prédominance des métiers du textile. Les petits métiers des rues représentent par ordre d'importance le deuxième groupe professionnel avec 13\% des incarcérées $»^{24}$.

Nous estimons que ces petits métiers, ceux des rues notamment, tout comme la prostitution occasionnelle, à titre de complément de ressources, sont constitutifs de l'identité de la roturière parisienne de Rétif. Ainsi si ces métiers ne fournissent pas au plus vieux métier du monde des recrues, ils entretiennent au moins un cheminement particulier vers la prostitution. Nous pensons particulièrement aux facilités de la promiscuité urbaine, aux trajets et aux stations dans l'espace des rues. C'est en ce sens que l'expression " courir les rues » s'est chargée d'une polysémie suspecte par analogie avec « les coureuses de bal $»^{25}$. Rétif cultive avec bonheur ce stéréotype dans ses Contemporaines. En effet, « courir les rues », expression redondante dans ses nouvelles qui renvoie généralement aux ouvrières exerçant leur métier semblerait un synonyme de « faire le trottoir ».

Ce grand expert de la prostitution met l'accent sur un autre fait dangereux qui ne cesse de favoriser ce commerce de chair. En effet, Rétif a montré comment la misère rassemble les âges et les sexes, pêle-mêle, dans des chambres étroites et provoque ainsi le libertinage par le rapprochement. Il donne l'exemple de l'industrie des grands ateliers qui continue cette incessante provocation pendant les heures de travail et pendant les allées et venues à la fabrique. Dans ce contexte, l'économiste Eugène Buret pense que

aucun enseignement moral, aucune surveillance, ne s'opposent à la corruption des mœurs chez les classes pauvres, obligées d'aller demander le pain chaque jour aux manufacturiers : le vice seul a la parole dans les ateliers, et la décence en est proscrite dans le langage et dans les actes, comme un ridicule [...] Dans cette atmosphère impure, $[\ldots]$ la jeune fille envisage la prostitution sas dégoût et sans effroi, comme une ressource contre la misère ${ }^{26}$

Dans tout cela, la prostitution apparaît comme fille légitime de la pauvreté. Dans ce contexte, Daniel Roche et Pierre Gourbet pensent 
qu'une « réflexion sur la pauvreté rassemble tous les débats du siècle, le luxe, la ville dévoreuse des hommes, la responsabilité sociale des élites et de l'Etat, la montée d'un libertinage sans borne dans les classes ouvrières $\gg{ }^{27}$. Nullement exclusif de la prostitution, le travail pourrait jouer un rôle incitateur notamment à cause de la faiblesse des salaires et la pénibilité de quelques métiers.

Nous estimons que ces ouvrières suspectes, que l'on glisse peu à peu dans la classe des prostituées sont la vraie facette de la marginale non pas au sens de clocharde mais dans le sens qu'elles n'ont pas une appartenance sociale stable. En effet, elles ne sont pas de vrais sujet sociaux, elles sont à la frontière entre l'inhumain et le social. Pourrions-nous parler ici d'un être hybride qui joigne humanité et animalité, vu qu'on les qualifie souvent de $«$ bêtes $»^{28}$.

Ces femmes vivent une double marginalité, la première dans leur premier métier et la deuxième dans le milieu prostitutionnel. Elles sont à mi-chemin entre les deux ne pouvant s'identifier totalement ni dans l'un ni dans l'autre.

Le souci documentaire dans Les Contemporaines du commun est très manifeste. Rétif écrivit à propos de cet ouvrage : «Un jour, ces nouvelles seront précieuses pour la postérité, parce qu'on y verra l'histoire du temps où vivait l'auteur $»^{29}$. C'est ainsi que Érica-Marie Benabou déclare ne pas pouvoir s'en passer de l'usage historique de ce texte. En effet, dans ces séries de nouvelles, Rétif manifeste un souci de décrire à la manière d'un guide vivant, pour aider à la compréhension du monde parisien. On y trouve les lieux communs de la représentation urbaine : les embarras de Paris et la découverte de mille petits métiers de la rue avec leurs figures obligées, la laitière du matin, la petite fruitière, la crieuse des chapeaux... tous un peu filous. Les petits métiers servent donc chez Rétif de fondement à une nomenclature des types humains et sociaux dont les prostituées constituent la classe dominante.

Rétif insinue à travers ses écrits qu'il est temps de parler des prostituées autrement que pour s'extasier devant leur charme et décolleté. Il y a de la misère et la souffrance derrière cela. Ce réformateur accuse ouvertement le système qui ouvre à la misère de la fille des villes, à l'aveuglement de la paysanne, la ressource funeste d'un travail exterminateur et la proximité des manufactures et du monde de la rue, qui les poussent peu après au vice et à la débauche. Michelet, en parlant du statut de l'ouvrière à cette époque, disait que c'est « la barbarie de notre occident [...] L'ouvrière ! mot impie, sordide, qu'aucune langue n'eut jamais, qu'aucun temps n'aurait compris avant cet âge de fer, et qui balancerait à lui seul tous nos prétendus progrès $»^{30}$. 


\section{Notes}

1. Nicolas-Edme Rétif de La Bretonne voit le jour à la campagne bourguignonne, précisément dans le village de Sacy en 1734, dans une famille de paysans quasiment cossus. Après une enfance quasiment pysanne, il devient ouvrier typographe à Auxerre ou il mène une vie agitée et quelque peu libertine. Il finit par s'installer à Paris jusqu'à son décès en 1806. Autodidacte, vivant dans un milieu très populaire et lié à l'humble monde des ouvriers et artisans, Rétif se distingue totalement des élites intellectuelles traditionnelles. Il était surtout connu comme auteur marginal à caractère ombrageux et instable. Ses contemporains retiennent surtout de lui sa grande fréquentation des filles de joie, auxquelles il a réservé une grande place dans son œuvre gigantesque.

2. Scarlett Beauvalet-Boutouryrie, Les femmes à l'époque moderns $\left(X V I^{e}-X V I I I^{e}\right)$, Paris : Belin, 2003, p. 232.

3. Scarlett Beauvalet-Boutouryrie, Histoire de la sexualité à l'époque moderne, Paris : Ibid, p. 274.

4. Louis-Sébastien Mercier, Le Tableau de Paris, éd. par Jean-Claude Bonnet, Paris : Mercure de France, 1994, t. II, p. 241

5. Beauvalet-Boutouryrie, op.cit., p. 233.

6. Rétif de La Bretonne, Monsieur Nicolas, éd. par Pierre Testud, Paris : Gallimard, 1989, t. II, p. 760.

7. Philip Schlesinger, «Essai sur l'ethnographie littéraire », Questions de communication [En ligne], 6 | 2004, mis en ligne le 06 octobre 2015, consulté le 02 décembre 2016. URL : http://questionsdecommunication.revues.org/4471; DOI : 10.4000/questionsdecommunication.4471.

8. Rétif de La Bretonne, Les Contemporaines du commun ou aventures des belles marchandes de l'âge présent, Paris : Les Yeux Ouverts, 1962, p. 2.

9. Alexandre Parent-Duchâtelet, La Prostitution à Paris au XIXe siècle, texte présenté par Alain Corbin, Seuil, 1981, p. 91-92.

10. Rétif de La Bretonne, Les Contemporaines du commun, « La jolie gazière », éd. par J. Assezat, Paris : Flammarion, 1873, p. 70.

11. Ibidem.

12. Ibid. p. 188.

13. Érica-Marie Benabou, La prostitution et la police des mœurs au XVIII ${ }^{e}$ siècle, Perrin, Paris, 1987, p. 288.

14. Les Contemporaines du commun, op.cit., p. 200.

15. Néologisme de Rétif qui veut dire habit

16. Benabou, op.cit., p. 297. 
17. Vincent Milliot, Les Cris de Paris ou le peuple travesti : les représentations des petits métiers parisiens (XVI ${ }^{e}-X V I I I^{e}$ siècles), Publications de La Sorbonne 1995, p. 37.

18. Les Contemporaines du commun, op.cit, p. 212.

19. Archive Nationale, Y 11496, Papiers du commissaire Chesnon, 13 juin 1776.

20. Benabou, op.cit., p. 312.

21. Ibid. p. 313.

22. Pornographe op.cit, p. 71.

23. Milliot, op.cit., p. 36.

24. Ibidem.

25. Type de prostituées dans « La jolie chirurgienne », une nouvelle des Contemporaines de Rétif.

26. Eugène Buret, La misère des classes laborieuses en Angleterre et en France, Ed Paulin, Lausanne, 1840, p. 492.

27. Pierre Goubert et Daniel Roche, Les Français et l'Ancien Régime, Ed Armand Paris : Colin, 1991, p. 99.

28. Expression répétée à maintes reprises par Rétif dans Le PalaisRoyal.

29. Rétif de La Bretonne, Les Contemporaines du commun, nouvelle $X X I V$, p. 345.

30. Jules Michelet, La Femme, Ed Paris: Flammarion, 1981, p. 54. 